\title{
QUALIDADE DE VIDA, ANSIEDADE E DEPRESSÃO EM PACIENTES DA HEMATO-ONCO COM DOR CRÔNICA
}

\section{QUALITY OF LIFE, ANXIETY AND DEPRESSION IN HEMATO- ONCOLOGY PATIENTS WITH CHRONIC PAIN}

\section{Aline Cristina Antonechen', Maribel Pelaez Dóro²}

\section{RESUMO}

A dor apresenta-se como um dos principais sintomas de pacientes com câncer e quando se estende por longo período, pode gerar consequências negativas na qualidade de vida (QV) e estar associada a transtornos psíquicos. Sendo assim, este trabalho visa expor sobre a QV e prevalência de ansiedade e depressão em pacientes que apresentam dor crônica e são assistidos no Ambulatório de HematoOncologia e Quimioterapia do HC-UFPR. Foram abordados 52 pacientes, que responderam os seguintes instrumentos: Questionário Demográfico, Escala HAD e Questionário da WHOQOL-Bref. Observou-se que, na QV, 40\% ( $n=21)$ obtiveram porcentagem menor que 50\% no domínio físico, $13 \%(n=7)$ no psicológico e de relações sociais e $8 \%(n=4)$ no de meio ambiente. A prevalência de ansiedade e depressão foram, respectivamente, de $46 \%$ e $28 \%$. Concluiu-se que a dor interfere na QV e na incidência de sintomas ansiosos e depressivos da população estudada, sendo necessário enfoque multidimensional para sua maior compreensão.

Descritores: Dor Crônica; Qualidade de Vida; Ansiedade; Depressão; Câncer
${ }^{1}$ Graduada em Psicologia pela Universidade Estadual de Maringá (UEM), Maringá, PR, Brasil.

${ }^{2}$ Doutora em Ciências da Saúde pela Universidade Federal do Paraná (UFPR), Curitiba, PR, Brasil.

\begin{abstract}
Pain presents as one of the main symptoms of cancer patients and when it extends for a long or permanent period it can bring negative consequences in quality of life and also be associated to anxiety and depression disorders. Regarding this context, the present work had the objective to demonstrate the quality of life and prevalence of anxiety and depression in patients with chronic pain who clinically assisted in Hemato-Oncology and Chemotherapy Ambulatory from Clinical Hospital of Federal University of Parana (HC-UFPR). Were approached 52 patients who responded the following instruments: demographic questionnaire, the Hospital Anxiety and Depression Scale (HAD) questionnaire and the World Health Organization Quality of Life (WHOQOL). In concerning quality of life, $40 \%(n=21)$ obtained result lower than $50 \%$ in physical domain; $13 \%(n=7)$ in psychological and social relations; and $8 \%(n=4)$ in environment. The prevalence of anxiety and depression were, respectively, $46 \%$ and $28 \%$. In conclusion, pain interferes in quality of life and in the incidence of anxious and depressive symptoms. Biological aspects cannot be the only focus, it is necessary to consider a multidimensional approach.
\end{abstract}

Descriptors: Chronic Pain; Quality of Life; Anxiety; Depression; Cancer. 


\section{Introdução}

$\underline{\text { Dor }}$

A dor é definida, pela Internacional Association for the Study of Pain (IASP) como "uma sensação ou experiência emocional desagradável, associada com dano tecidual real ou potencial"'. Essa sensação é classificada de acordo com alguns critérios, dentre eles, a duração, podendo ser considerada crônica ou aguda. Esta é definida pelo curto período de duração (de horas a semanas) e pela função de alerta, sinalizando que o organismo encontra-se em perigo por algum motivo².

Já a dor crônica pode durar de meses a anos, sendo associada a lesões já diagnosticadas e em tratamento e a doenças crônicas ${ }^{2,3,4}$. Pode afetar diversas dimensões da vida do indivíduo, ocasionando em prejuízos para a qualidade de vida e associarse a transtornos de humor ${ }^{5,6}$. Estudos apontam que a prevalência de dor crônica pode variar de $12 \%$ a $80 \%$, de acordo com as variáveis consideradas ${ }^{4}$. Em todo mundo atinge cerca de 100 milhões de pessoas, ou seja, $20 \%$ a $40 \%$ da população em geral ${ }^{8,9}$.

No que concerne ao câncer, um estudo da Organização Mundial da Saúde (OMS) apontou que 5 milhões de pessoas em todo o mundo apresentam dor de câncer e $25 \%$ delas morre sem alivio ${ }^{12}$. Estudos revelaram que a dor está presente em $35-45 \%$ dos pacientes à data do diagnóstico e em $70-90 \%$ quando a doença está avançada ${ }^{10,11}$. Essa pode ser associada à doença ou ser secundária ao tratamento realizado. Demonstrou-se que $60-90 \%$ dos casos a dor está relacionada à neoplasia, em 5-20\% ao tratamento e apenas em 3-10\% dos casos não há relação entre a neoplasia e a dor ${ }^{10,11}$.

A percepção de dor pode ser aumentada por conta de outros sintomas que também acometem o paciente com câncer, como a falta de apetite, alterações de sono, náuseas, dentre outros. Somado a isso, o estado emocional desses pacientes também pode apresentar-se abalado, causando alterações na maneira como eles percebem e simbolizam a vida, inclusive a dor. Assim, ressalta-se a importância da realização de uma avaliação integral do paciente por profissionais de diferentes áreas, para controle da dor e do sofrimento que 0 aflige e consequente melhoria de sua qualidade de vida ${ }^{12,13}$.

Quando a dor é compreendida além de seus aspectos fisiológicos, revela-se enquanto uma Dor Total. Esse termo foi cunhado por Dame Cecily Saunders e abarca também os fatores psicológicos, sociais e espirituais relacionados a essa sensação ${ }^{14}$. A terminologia foi utilizada inicialmente para a dor sentida por pacientes terminais, contudo percebeuse que pode ser aplicada para casos de dor crônica, em que são relatados aspectos mais abrangentes que apenas o fisiológico. Essa compreensão nos fornece uma visão holística de ser humano, ou seja, necessita ser tratado não somente em seu corpo, mas também em seus valores e essênciaa-14,15.

\section{Qualidade de vida}

A qualidade de vida (QV) é definida pela Organização mundial de Saúde (OMS) como "percepção do indivíduo de sua posição na vida, no contexto da cultura e sistema de valores nos quais ele vive e em relação aos seus objetivos, expectativas, padrões e preocupações"16. Assim sendo, os indicadores de QV se alteram para cada indivíduo e estará de acordo com as percepções que este tem de suas vivências ${ }^{17}$.

Em pacientes com enfermidades crônicas, como o câncer, a QV vem sendo mensurada a fim de direcionar a prática clínica realizada pelos profissionais. A convivência com uma doença crônica impõe a muitos enfermos períodos difíceis, com dor, estresse, ansiedade, dentre outros sintomas, apesar dos tratamentos ofertados ${ }^{13}$. Compreender a maneira como esses sintomas influenciam em diferentes aspectos da vida do paciente pode auxiliar para que se tenha uma prática que leve em consideração a real demanda trazida por ele.

Estudos sobre a QV em pacientes com câncer demonstram que esta é influenciada por diversos fatores, como o tipo de câncer, o estágio da doença, presença/ausência de metástases, o tratamento realizado, a sintomatologia, dentre outros ${ }^{19,20,21,22}$.

Um dos sintomas que interferem na QV é a dor. Pesquisas apontam que pacientes que apresentam essa sensação tem alterações significativas de QV, principalmente no domínio físico ${ }^{18-24}$. Portanto, pode-se aferir que a dor enquanto sintoma único apresenta-se como um fator de influência na QV. Associada aos demais sintomas, torna-se um fator de risco a ser considerado. 


\section{Ansiedade e depressão}

Segundo o CID-10, o transtorno depressivo caracteriza-se por alguns sintomas como humor deprimido, perda de interesse em atividades antes consideradas agradáveis e energia diminuída ${ }^{24}$. O DSM-5 pontua outros sintomas também, como perda ou ganho de peso, insônia ou hipersonia, sentimento de culpa ou inutilidade, entre outros ${ }^{25}$. A etiologia dessa situação clínica é multifacetária, podendo estar relacionados a fatores biológicos, psicossociais e genéticos.

Já o transtorno de ansiedade é descrito no CID-10 como tensão, preocupação e sentimento de apreensão sobre eventos e problemas cotidianos e apresenta sintomas físicos, mentais e tensão $0^{24}$. O DSM-5 postula que nesse transtorno há ansiedade e preocupação excessiva na maioria dos dias por pelo menos seis meses, difícil controle da preocupação, sintomas físicos, que essa preocupação cause sofrimento e que não esteja associado a uso de substancias ou outros transtornos mentais ${ }^{25}$.

Em pacientes com câncer, a depressão está relacionada principalmente à piora na evolução do estado clínico e baixa qualidade de vida. 0 diagnóstico desse quadro, no entanto, nem sempre é fácil e preciso e há diversas hipóteses quanto a sua etiologia e consequência para os pacientes. $O$ tratamento mais indicado e que vem apresentando eficácia é o farmacológico associado ao psicológico ${ }^{13}$.

As alterações e necessidade de adaptação a um novo contexto de vida e o medo da morte também podem gerar no paciente com câncer sentimentos de angústia. Como consequência, pode haver dificuldades de compreensão e aceitação em relação à enfermidade e ao tratamento, além de uma piora da qualidade de vida. A forma de tratamento será de acordo com o motivo gerador de ansiedade, podendo ser necessário intervenção farmacológico, psicológica e social, dentre outros ${ }^{13}$.

Na população em geral, a prevalência de ansiedade é de aproximadamente $16 \%$ e de depressão, em torno de $16,2 \%{ }^{27}$. Nas doenças médicas, o predomínio de depressão é entre 15\% e 61\% e de ansiedade, entre 18\% e 35,1\%, sendo a discrepância desses valores relacionada às diferentes formas de avaliar essas duas reações ${ }^{27}$. Em pacientes com câncer, foi encontrado que $25 \%$ daqueles que estão em cuidados paliativos apresentam depressão e $73 \%$ tem sintomas de ansiedade ${ }^{28}$.

A ansiedade e a depressão também podem relacionar-se à dor. $O$ paciente ansioso pode ter uma percepção aumentada de sua dor e em contrapartida, a dor pode ser entendida como uma forma de defesa do indivíduo, o que faz com que seu sistema entre em alerta e assim, haja ansiedade. Alguns dos tratamentos possíveis serão os fármacos, esclarecimentos ao paciente, psicoterapia, técnicas de relaxamento e hipnose 2 .

Quanto a depressão, é comumente encontrada no contexto médico, porém muitas vezes subdiagnosticada e até mesmo confundida com outras patologias. 0 paciente que apresenta dor pode entrar em estado depressivo, principalmente quando essa dor tem uma intensidade significativa e duração de meses e até anos e interfere de forma abrupta nas vivencias do indivíduo. Para tratamento, pode ser necessária intervenção farmacológica e psicológica. 0 manejo da dor também é uma maneira indireta de tratar a depressão².

Nesse contexto, este artigo tem por objetivo expor sobre a QV e a prevalência de ansiedade e depressão em pacientes que apresentam dor crônica e são atendidos em um ambulatório de hemato-oncologia e quimioterapia de um hospital escola.

\section{Metodologia}

Este estudo é uma pesquisa clínica quantitativa, transversal, prospectiva e randomizada. Para este estudo não houve conflito de interesses.

A coleta de dados foi realizada no período de abril a julho de 2014, após aprovação do projeto pelo Comitê de Ética em Pesquisa (CEP) (CAAE: 27443914.2.0000.0096). Por ser um estudo randomizado, foram abordados os cinco primeiros pacientes que compareceram ao Ambulatório de Hemato-Oncologia e Quimioterapia do Hospital de Clínicas da Universidade Federal do Paraná (HC-UFPR). Os pacientes eram abordados em sala de espera e a aplicação dos questionários foi realizada nesse local, em um espaço com menor fluxo de pessoas.

Para que o paciente fosse convidado a participar do estudo, deveria responder aos seguintes critérios: Ter idade igual ou superior a 18 anos; responder aos critérios determinados para dor crônica; ser paciente do Ambulatório de Hemato-Oncologia e Quimioterapia; apresentar condições cognitivas para compreender o teor dos questionários e; aceitar assinar o Termo de Consentimento Livre e Esclarecido (TCLE). 
Para determinar se o paciente era portador de dor crônica, foram utilizados os critérios preconizados pela Portaria n 1083 do Ministério da Saúde, uma vez que, este estudo está de acordo com a concepção de dor crônica referendada nessa Portaria. A pesquisadora perguntava ao paciente se a dor dele tinha duração igual ou superior a 30 dias, com intensidade mensurada pela Escala Visual Analógica (EVA) acima de 4 e/ou se tomava medicamentos para controle de dor que se enquadrassem na Escala Analgésica da OMS.

Como instrumentos para coleta de dados, foram utilizados os seguintes protocolos e escalas: um questionário clínico demográfico, a fim de levantar o perfil sociodemográfico e clinico da população estudada; Escala Hospitalar de Ansiedade e Depressão (HAD) para averiguar a taxa de ansiedade e depressão e; questionário da World Health Organization Qualityof Life (WHOQOL) para mensurar a qualidade de vida. Tendo em vista que o questionário original da WHOQOL consiste em 100 questões, exigindo tempo prolongado para aplicação, foi utilizada sua versão abreviada (WHOQOL-bref) que conta com somente 26 questões.

Foram realizadas 208 abordagens e destas 52 enquadraram-se nos critérios para inclusão na pesquisa. Os principais motivos para não inclusão foram: não sentir dor, ter sido abordado anteriormente e a intensidade da dor ser relatada como abaixo de 4 na EVA.

Para a análise de dados, foram realizadas algumas relações entre as variáveis existentes nos instrumentos utilizados. Foi utilizada uma análise descritiva em que pode se observar as correlações e dispersões. Além disso, foram calculados os coeficientes de correlação de Pearson entre as variáveis de interesse e os quatro domínios de qualidade de vida estudados.

\section{Resultados}

Dos 52 participantes, $79 \%$ ( $n=41$ ) eram do gênero feminino, com idade média de 54,8 anos, variando entre 22 e 86 anos e $65 \%$ ( $n=34$ ) mantinham união estável (casados, vivendo como casados ou em relacionamento sério).

Dentre essa população, $59 \%$ ( $n=31$ ) tinham menos de 8 anos de estudos e $5,8 \%$ ( $n=3$ ) estavam cursando universidade, sendo os cursos eleitos: administração, enfermagem e jornalismo. 39\% ( $n=20)$ apresentavam vínculo empregatício, porém destes somente $53 \%$ ( $n=17$ ) estavam trabalhando, o restante encontrava-se com licença doença ou afastado. Das profissões referenciadas, $88 \%(n=15)$ diziam respeito a trabalhos braçais, como diarista, auxiliar de serviços gerais, agricultor, dentre outros.

Desses pacientes, $67 \%(n=35)$ apresentavam comorbidades, como demonstrado na tabela abaixo:

Tabela 1 - Comorbidades apresentadas pelos 52 participantes da pesquisa:

\begin{tabular}{c|c|c}
\hline Comorbidade & n. de pacientes portadores & \% total de pacientes portadores \\
\hline Hipertensão/hipotensão & 14 & $27 \%$ \\
\hline Diabetes & 9 & $17 \%$ \\
\hline Artrose & 8 & $15 \%$ \\
\hline $\begin{array}{c}\text { Doenças relacionadas aos } \\
\text { ossos }\end{array}$ & 7 & $13 \%$ \\
\hline Tireoidismo & 5 & $10 \%$ \\
\hline $\begin{array}{c}\text { Dificuldades visuais e } \\
\text { auditivas }\end{array}$ & 3 & $6 \%$ \\
\hline Dificuldades respiratórias & 3 & $6 \%$ \\
\hline $\begin{array}{c}\text { Doenças circulatórias } \\
\text { Colesterol alto }\end{array}$ & 3 & $6 \%$ \\
\hline $\begin{array}{c}\text { Cistos e tumores, } \\
\text { inflamação e osteoporose }\end{array}$ & 3 & $2 \%$ \\
\hline $\begin{array}{c}\text { Insônia, depressão, } \\
\text { fibromialgia, epilepsia, } \\
\text { lúpus, cirrose, anemia } \\
\text { crônica, dor de cabeça } \\
\text { crônica e pedra na vesícula }\end{array}$ & 2 & \\
\hline
\end{tabular}


Os tipos de doenças oncológicas e hematológicas mais encontrados são especificados na tabela 2:

Tabela 2 - Doenças hematológicas/oncológicas apresentadas pelos 52 participantes da pesquisa

\begin{tabular}{c|c|c}
\hline Diagnóstico & n. de pacientes portadores & \% total de pacientes portadores \\
\hline Neoplasia de mama & 16 & $30 \%$ \\
\hline $\begin{array}{c}\text { Transtornos de sangue e } \\
\text { doenças hematopoéticas }\end{array}$ & 12 & $23 \%$ \\
\hline Linfoma Não-Hodgkin & 9 & $17 \%$ \\
\hline Mieloma Múltiplo & 3 & $6 \%$ \\
\hline Imunodeficiência Comum & 1 & $2 \%$ \\
\hline Variável & 1 & $2 \%$ \\
\hline Linfoma Hodgkin & 1 & $2 \%$ \\
\hline Neoplasia de cólon & 1 & $2 \%$ \\
\hline Neoplasia de fígado & 1 & $2 \%$ \\
\hline Neoplasia de esôfago & 1 & $2 \%$ \\
\hline $\begin{array}{c}\text { Neoplasia maligna sem } \\
\text { especificação de localização }\end{array}$ & 1 & $2 \%$ \\
\hline Neoplasia de ovário & 1 & $2 \%$ \\
\hline Neoplasia de próstata & 1 & $\mathbf{2} \%$ \\
\hline Neoplasia de rim & 1 & $2 \%$ \\
\hline Neoplasia de timo & 1 & \\
\hline Osteossarcoma & 1 & $2 \%$ \\
\hline Sarcoma de Kaposi & $\mathbf{5 2}$ & \\
\hline Total & 100 & \\
\hline & 1 & $2 \%$ \\
\hline
\end{tabular}

O tempo médio de dor foi de 90 meses e a intensidade média da dor mensurada pela EVA atingiu 7 pontos. A dor foi associada ao câncer em $31 \%(n=16)$ dos casos. Os pacientes que tomavam medicamento para controle da dor representaram $88 \%(n=46)$ da amostra e $50 \%(n=23)$ destes tomavam apenas um medicamento, sendo predominante a monoterapia com analgésicos.

Dos pacientes que tomavam medicamento, $39 \%(n=18)$ seguiam a escala analgésica de dor da OMS, sendo que $50 \%(n=9)$ deles estavam no degrau 1 (analgésicos e anti-inflamatórios + fármaco adjuvante), 33\% ( $n=6)$ no degrau 2 (analgésicos e anti-inflamatórios + fármaco adjuvante + opióides fracos) e 17\% ( $n=3$ ) no degrau 3 (analgésicos e antiinflamatórios + fármaco adjuvante + opióides fortes). E ainda daqueles que tomavam medicamentos, $11 \%$ afirmaram não saber ou não se recordar o nome do medicamento utilizado.

Quanto às técnicas complementares e recursos, $60 \%$ dos participantes afirmaram uso terapêutico para decréscimo da dor. Foram utilizados tanto recursos e técnicas passivos, que não requerem esforço por parte do paciente, como ativos, que necessitam de uma participação ativa.As técnicas e recursos passivos mencionados foram acupuntura, massagens, cinta de mobilização, chá, ficar deitado, escutar música, bolsa de água quente e escalda pés. Quanto às técnicas e recursos ativos, foram citados a fisioterapia, exercícios físicos e a respiração.

Em relação à qualidade de vida, $40 \%$ ( $n=21)$ obtiveram porcentagem menor que $50 \%$ no domínio físico, 13\% $(n=7)$ no domínio psicológico e de relações sociais e $8 \%(n=4)$ no domínio de meio ambiente. Em contrapartida, foram constatadas porcentagens maiores de $80 \%$ entre $13 \%(n=7)$ dos participantes no domínio físico, $33 \%$ ( $n=17$ ) no domínio psicológico, 38\% ( $n=20)$ no domínio de relações sociais e 36\% ( $n=19)$ no domínio de meio ambiente.

Por fim, indícios de depressão possível foram apresentados por 15\% ( $n=8)$ dos participantes e provável em 13\% $(n=7)$. E a ansiedade foi considerada possível para $31 \%(n=16)$ dos pacientes e provável em 15\% (n=8). 20\% $(n=10)$ apresentaram ambos os transtornos de humor. 


\section{Discussão}

Segundo dados encontrados, a dor apresenta maior prevalência e intensidade entre as mulheres. Há estudos que já apontam essa diferença e a correlacionam a diversos fatores biopsicossociais ${ }^{4-31,32}$, sendo um desses a questão financeira. Quando a dor afeta na capacidade de executar atividades laborais, o homem é mais prejudicado do que a mulher, uma vez que seu papel social tende a estar associado ao trabalho e ao sustento da família. Consequentemente, aquele pode demonstrar maior tolerância, uma vez que as percas suscitadas lhe causam mais sofrimento do que a dor em si.

Além disso, de acordo com a definição de dor fornecida pelos participantes, há uma ligação direta entre essa e o sofrimento, que é predominantemente expresso pelo gênero feminino. No contexto sociocultural vigente, o espaço destinado para manifestação de emoções faz-se mais presente no meio feminino e uma das linguagens simbólicas utilizadas para demonstrar o sofrimento é a dor, tendo em vista que nem sempre a expressão verbal é valorizada ou consegue ser acessada.

No que concerne à Qualidade de Vida, foi considerada boa no grupo estudado, já que mais da metade dos participantes obtiveram pontuações maiores que 50\% nos domínios analisados.

O domínio mais prejudicado foi o físico e infere-se que um dos fatores de influência foi a faixa etária, pois $44 \%$ ( $n=23$ ) dos entrevistados apresentavam idade superior a 60 anos. Durante a entrevista, foi observado que algumas das queixas relatadas quanto aos aspectos físicos estavam relacionados às limitações advindas do avanço da idade e comorbidades associadas.

No domínio psicológico, a idade também se mostra como fator de interferência, devido à representação social do envelhecimento disseminada na sociedade ocidental contemporânea. Estar na terceira idade, apesar de ser dita a melhor idade, pode representar perdas e declínio de todas as funções, havendo consequente desvalorização dessa etapa da vida. Quando essa ideia é interiorizada pelo idoso, há sentimentos de autoestima baixa, inutilidade e incapacidade, o que pode influenciar no processo de adoecimento dessa população. Estudos citam, inclusive, que o declínio cognitivo naqueles que se encontram em idade avançada está mais relacionado a outros fatores do que com o envelhecimento em $\mathrm{si}^{33}$.

Segundo análise estatística através da Correlação de Pearson, duas variáveis apresentaram-se significativamente relevantes quando associadas ao domínio psicológico, que foram o tempo de dor ( $p$-valor: 0,950) e a depressão ( $p$-valor: 2,96). A doença e a dor crônica expõem o individuo a uma série de limitações e sensações desagradáveis em seu curso, sendo associadas, assim, ao sofrimento psíquico. Tendo em vista que a experiência de dor é particular e subjetiva, saber qual papel que ela ocupa na vida daquele que a vivencia é fundamental para compreender sua relação com o sofrimento. Também é relevante identificar os fatores causadores dessa dor, os elementos que favorecem a tolerância e os fatores de enfrentamento que cada indivíduo encontra nessa situação, para auxiliar na melhora de sua qualidade de vida e diminuir o possível impacto psicológico ocasionado por essa sensação ${ }^{13}$.

O sofrimento relacionado à dor crônica também pode ser fator de risco para o desenvolvimento de transtornos depressivos. A porcentagem de depressão possível ou provável ( $28 \%$ dos entrevistados) encontrada nesse estudo foi considerada dentro dos parâmetros esperados para aqueles indivíduos com doenças médicas, que é de $15 \%$ a $61 \%$ da população.

O tempo prolongado de exposição a uma sensação desagradável como a dor pode alterar a maneira como o indivíduo percebe a sua vida, adquirindo pensamentos negativos e falta de motivação. Dessa forma, a depressão torna-se uma doença secundária à dor e resulta em piora da qualidade de vida daquele que a vivencia². Outra perspectiva é que a depressão gera um posicionamento introspectivo no deprimido, que se volta para seu mundo interno. Assim, qualquer sensação tem relevância devido a maior sensibilidade, que em condições saudáveis seria imperceptível. No caso da dor, o transtorno depressivo pode fazer com que o paciente esteja mais atento a sensação desagradável e a perceba commagnitude ${ }^{34}$.

Outros dados que apresentaram relevância significativa foram os cruzamentos entre o domínio de relações sociais e o tempo de dor ( $p$-valor: 0,384 ); desse mesmo domínio com a depressão ( $p$-valor: 2,81) e; a relação entre o domínio de meio ambiente com o tempo de dor ( $p$-valor: 0,461). A doença, a dor por tempo prolongado e a idade avançada são fatores que isoladamente já causam impacto biopsicossocial e quando associadas agravam ainda mais a qualidade de vida global. Tais fatores limitam a interação do indivíduo com o seu meio, tanto por suas questões intrínsecas como extrínsecas.

Quando o corpo físico padece, há reverberação para o corpo psíquico, ou seja, a percepção que a pessoa tem de si também é alterada. Em consequência, se não há satisfação com a imagem que se apresenta, o indivíduo tende a retrair-se e provocar o isolamento social e a diminuição da interação com o meio em que se está inserido ${ }^{35}$. Apresenta-se assim, uma relação direta entre os fatores intrínsecos do domínio de relações sociais e o estado emocional e psíquico em que a pessoa se encontra. Essa afirmação é ratificada pela associação evidente entre a depressão e o prejuízo no domínio de relações sociais. 
Quanto aos fatores extrínsecos estão os limites impostos pela condição física, que impedem aquele que padece de exercer suas atividades sociais, tais como trabalhar, reunir-se com amigos e familiares, frequentar os lugares que Ihe traziam satisfação, dentre outros ${ }^{14}$. $O$ indivíduo pode passar grande parte de seu tempo em casa ou em instituições de saúde, na qual seu contato é restrito aos familiares mais próximos e profissionais de saúde. Há, dessa maneira, uma readequação do papel social deste perante a sociedade, ou seja, deixa de ser o trabalhador, provedor da família, cuidador do lar, para tornar-se o paciente, aquele que necessita de cuidados.

Por fim, no que condiz à ansiedade, foi constatada em quase metade da população estudada (46\% dos entrevistados), mas não apresentou relação significativa com nenhum dos domínios da qualidade de vida e nem mesmo com o tempo de dor. Ao comparar com a prevalência desse transtorno em pacientes com doenças médicas, esse número se encontra acima da média, que seria entre $18 \%$ e $35,1 \%$ dos pacientes.

Dentre algumas perspectivas, o transtorno de ansiedade pode ser compreendido como uma resposta emocional do indivíduo frente a uma situação desconhecida e/ou percebida como ameaçadora e está ligado a uma antecipação do futuro. $O$ medo desse porvir gera sentimentos de angustia que nem sempre são facilmente identificados e por vezes podem ser somatizados através de sensações corporais, tais como a dor². Uma investigação detalhada do discurso do paciente, principalmente quando há sintomas de ansiedade evidentes, podem deflagrar que sua sintomatologia está mais relacionada a seu funcionamento psíquico e a angustia latente do que necessariamente com a doença.

\section{Considerações Finais}

Apesar da qualidade de vida de mais da metade dos entrevistados estar acima da média de porcentagem, há prejuízo em alguns domínios, principalmente quando correlacionados com a ansiedade e depressão. 0 domínio mais prejudicado foi o físico, que diz respeito a percepção de dor, fadiga, sono, necessidade de tratamento médico e capacidade de locomoção e de exercer atividades diárias. Entretanto a maior correlação se deu entre o domínio psicológico e a depressão.

Pelo relato dos participantes, a dor crônica influenciou na qualidade de vida e sintomas de ansiedade e depressão, demonstrando que essa sensação interfere não somente no sistema biológico, mas também no psíquico, social e espiritual do indivíduo. Assim sendo, corrobora-se com o conceito de Dor Total e se valida a hipótese de que o sofrimento ocasionado pela situação em que o paciente se encontra é também qualificado em termos de dor.

É fundamental para o bem-estar global do paciente que o mesmo receba um tratamento originário do entendimento advindo de estudos científicos, mas, também de reflexões da prática clínica que englobem o cuidado integral. Posto isto, compreende-se que envolve um conhecimento técnico-científico, mas, o que norteia o cuidado e o trabalho a ser desenvolvido é a ética. Sendo assim, os profissionais de acordo com cada especialidade e especificidade de conduta terapêutica deve considerar a doença, o tratamento prescrito e o doente que tem dor e sofre no seu contexto de inserção. Para tanto, é premente a escuta qualificada da clínica como um todo, por ser capaz de discernir, interpretar e integrar os valores objetivos/subjetivos expressos pelo paciente. Nesse sentido, a compreensão do significado da dor e a sua maior ou menor interferência na vida daquele que adoece pode auxiliar na busca de tomada de decisões, soluções, acolhimentos e tratamentos mais efetivos e eficazes.

\section{Referências}

1. BRASIL. Ministério da Saúde. Portaria n. 1083, de 02 de outubro de 2012. Aprova o Protocolo Clínico e Diretrizes Terapêuticas da Dor Crônica. Brasília, DF, 02 out., 2012

2. Camon VAA. Psicossomática e a Psicologia da Dor. $2^{\mathrm{a}}$ ed. São Paulo: Pioneira Thomson Learning, 2012.

3. Dellaroza MSG, Pimenta CAM, Lebrão ML, Duarte YAO, Braga PE. Associação entre dor crônica e autorrelato de quedas: estudo populacional - SABE. Cad. Saúde Pública 2014; 30 (3): 522-32 .

4. Vieira EBM, Garcia JBS, Silva AAM, Araújo RLTM, Jansen RCS, Bertrand ALX. Chronic pain , associated factors, and impact on daily life: are there differences between the sexes? Cad. Saúde Pública 2012;28(8): 1459-67.

5. Hennemann-Krause L. Dor no fim da vida: Avaliar para tratar. Rev. do Hosp. Univ. Ernesto Pedro UERJ 2012; 11 (2): 26-31. 6. Sleutjes A. Relação entre dor crônica, atenção e memória: [s.n.], 2011. 144 p. Dissertação (Mestrado) - Faculdade de Medicina da Universidade de São Paulo, 2011. 
7. Lima MAG, Trad LAB. "Circuloterapia": uma metáfora para o enfrentamento da dor crônica em duas clínicas de dor. Rev. Saúde Coletiva 2011; 21 (1): 217-36.

8. Almeida JG, Kurita GP, Braga PE, Pimenta CAM. Dor crônica em pacientes esquizofrênicos: prevalência e características. Cad. Saúde Pública 2010; 26 (3): 591-602.

9. Cunha LL, Mayrink WC. Influência da dor crônica na qualidade de vida de idoso. Rev. Dor 2011; 12 (2): 120-4.

10. Costa CA, Santos C, Alves P, Costa A. Dor oncológica. Rev. Portuguesa de Pneumologia 2007; 13 (6): 855-67.

11. BRASIL. Ministério da Saúde. Instituto Nacional do Câncer. Cuidados Paliativos Oncológicos: controle da dor. Rio de Janeiro, INCA, 2001

12. Gallagher RM. Clínicas Médicas da América do Norte: Dor Crônica. Rio de Janeiro: Reichmann\& Affonso Editores, 1999. 13. Carvalho VA, Franco MHP, Kóvacs MJ, Liberato RP, Macieira RC, Veit MT, et. al. Temas em psico-oncologia. São Paulo: Summus Editorial, 2008.

14. Santos FS. Cuidados Paliativos: Discutindo a Vida, a Morte e o Morrer. São Paulo: Editora Atheneu, 2009

15. Capelas MLV. Dor Total nos doentes com metastização óssea. Cad.deSaúde, Lisboa 2008; 1 (1): 9-24.

16. WORLD HEALTH ORGANIZATION. WHOQOL: Measuring Quality of Life. Suiça, Geneva, 1997. Disponível em: <http://www.who.int/mental_health/media/68.pdf>

17. Pedrosa DFA, Pelegrin AKAP, Siqueira HBOM, Silva TCR, Colhado OCG, Souza FAEF. Avaliação da qualidade de vida em clientes com dor crônica isquêmica. Rev. Latino-Am. Enfermagem 2011; 19 (1): 1-6.

18. Mansano-Schlosser TC, Ceolim MF. Qualidade de vida de pacientes com câncer no período de quimioterapia. Texto \& Contexto Enfermagem 2012; 21 (3): 600-7.

19. Chaves PL, Gorini MIPC. Qualidade de vida do paciente com câncer colorretal em quimioterapia ambulatorial. Rev. Gaúcha de Enferm. 2011; 23 (4): 767-73.

20. Arrais T. A. Qualidade de Vida de mulheres usuárias do SUS com câncer de colo de útero, atendidas no hospital de Palmas-TO. Brasília: [s.n.], 2010, 73 p. Dissertação (mestrado) - Programa de Pós-Graduação em Ciências da Saúde da Universidade de Brasília, 2010.

21. Franceschini J, Jardim JR, Fernandes ALG, Jamnik S, Santoro IL. Relationship between the magnitude of symptons and the quality of life: a cluster analysis of lung cancer patients in Brazil. J. Bras. Pneumol. 2013; 39 (1): 23-31.

22. Andrade V, Sawada NO, Barichello E. Quality of life in hematologic oncology patients undergoing chemotherapy. Rev. Esc. Enferm. USP 2013; 47 (2): 350-6.

23. Queiroz MF, Barbosa MH, Lemos RCA, Ribeiro SBF, Ribeiro JB, Andrade EV, Silva QCG, et. al. Qualidade de vida de portadores de dor crônica atendidos em clínica multiprofissional. Rev. de enfermagem e atenção à saúde 2012; 1 (1): 30-43.

24. CID-10 - Critérios diagnósticos para pesquisa/Organização Mundial de Saúde; trad.: Maria Lúcia Domingos; - Porto Alegre: Artes Médicas, 1998

25. Referência rápida aos critérios diagnósticos do DSM-5 - Porto Alegre: Artmed, 2014

26. Bragança GMG. Avaliação da prevalência de ansiedade e depressão em pacientes com hiperidrose primária grave. Aracaju: [s.n.], 2012. 55 p. Dissertação (Mestrado) - Programa de Pós-Graduação em Saúde e Ambiente da Universidade Tiradentes, 2012.

27. Telles-Correia D, Barbosa A. Ansiedade e depressão em medicina: modelos teóricos e avaliação. Acta Med. Port. 2009; 22 (1): 89-98.

28. Júnior NCS. Depressão, ansiedade e qualidade de vida em mulheres em tratamento de câncer de mama. Brasília: [s.n.], 2010. 71 p. Dissertação (Mestrado) - Faculdade de Ciências da Saúde da Universidade de Brasília, 2010.

29. Hochman B, Nahas FX, Filho RSO, Ferreira LM. Desenhos de pesquisa. Acta. Cirúrgica Brasileira 2005; 20 (2).

30. Souza RF. $O$ que é um estudo clínico randomizado? Medicina (Ribeirão Preto) 2009; 1 (42): 3-8.

31. Nogueira M, Casetto SJ. Gênero e enfrentamento da dor. Psic. Rev. SãoPaulo 2006; 2 (15): 161-76.

32. Palmeira CCA, Ashmawi HA, Junior JOO, Posso IP. Opióides, sexo e gênero. Rev. Dor São Paulo 2011; 2 (12): $182-87$.

33. Schneider RH, Irigaray TQ. $O$ envelhecimento na atualidade: aspectos cronológicos, biológicos, psicológicos e sociais. Estudos de Psicologia 2008; 4(25): 585-93.

34. Pimenta CAM, Koizumi MS, Teixeira MJ. Dor crônica e depressão: estudo em 92 pacientes. Rev. Esc. Enf. USP 2000; 34 (1): 76-83.

35. Zimmermann E. Corpo e Individuação. São Paulo: Editora Vozes, 2009 


\section{Aline Cristina Antonechen}

Endereço para correspondência - Rua: Magda Perona Frossard, $n^{\circ} 255$, apto 901, Bairro: Nova Aliança, CEP: 14026-596, Ribeirão Preto, SP, Brasil.

E-mail: aline_antonechen@hotmail.com Lattes: http://lattes.cnpq.br/4820356769096513

Maribel Pelaez Dóro - maripdoro@hotmail.com

Enviado em 01 de agosto de 2015. Aceito em 18 de março de 2016. 
